\title{
Philosophiques
}

\section{Éthique et responsabilité - Paul Ricœur, textes réunis par J.-Ch. Aeschlimann Neuchâtel, la Baconnière, Coll. : " Langages ", 1994, 197 pages.}

\section{Daniel Desroches}

Volume 26, numéro 1, printemps 1999

URI : https://id.erudit.org/iderudit/004915ar

DOI : https://doi.org/10.7202/004915ar

Aller au sommaire du numéro

Éditeur(s)

Société de philosophie du Québec

ISSN

0316-2923 (imprimé)

1492-1391 (numérique)

Découvrir la revue

Citer ce compte rendu

Desroches, D. (1999). Compte rendu de [Éthique et responsabilité - Paul

Ricœur, textes réunis par J-Ch. Aeschlimann Neuchâtel, la Baconnière, Coll. :

" Langages », 1994, 197 pages.] Philosophiques, 26(1), 101-103.

https://doi.org/10.7202/004915ar d'utilisation que vous pouvez consulter en ligne.

https://apropos.erudit.org/fr/usagers/politique-dutilisation/ 


\section{Éthique et responsabilité - Paul RICÆUR, textes réunis par J.-Ch. Aeschlimann \\ Neuchâtel, la Baconnière, Coll.: « Langages », 1994, 197 pages.}

Ce livre « entend mettre en évidence quelques enjeux propres à l'œuvre et à la pensée de Paul Ricœur» (p. 7). Cet ouvrage collectif, construit autour d'un entretien mené par J.-Ch. Aeschlimann, réunit sept contributions auxquelles on a joint un échange épistolaire avec Emmanuel Lévinas. Il faut ajouter également que ce livre fait suite à un ouvrage collectif publié en 1989 en l'honneur de Lévinas lui-même, dont le titre était, chez le même éditeur, Répondre d'autrui.

L'entretien, qui porte sur la reconstruction de l'Europe après les événements de 1989 ainsi que sur les travaux philosophiques récents de l'auteur, fait d'emblée ressortir l'importance que Ricœur accorde à la mémoire : "La mémoire du terrible nous est absolument indispensable» (p. 20, cf. aussi p. 14, 15 et 19). L'opinion de Ricœur sur l'actualité européenne, où l'« effondrement du mensonge » qualifiant la fin du communisme occupe une place plus grande que l'essor de l'économie de marché, conduit la discussion à la problématique de l'identité présente dans Soimême comme un autre (Seuil, 1990). Des thèmes abordés, nous retenons les principaux : la primauté de l'éthique sur la morale, la promesse comme base de l'institution langagière, les composantes - ipse et idem - propres à l'identité personnelle et le rôle de l'identité narrative dans la constitution du sujet.

Certes, l'échange de lettres entre Lévinas et Ricœur ne surprendra personne. Le premier insiste sur l'irréductible injonction au "pour-l'autre » dont témoigne le visage, et à laquelle il rattacherait maintenant une "non-indifférence à soi ", celle d'une "dignité d'élu qui [...] s'énonce dans l'unicité humaine du pronom je » (p. 36). Quant au second, en accord avec l'unicité du pronom je, il soutient que l'interpellation par l'autre à la responsabilité ne saurait être reconnue telle sans une « estime de soi » capable de répondre, comme le veut Lévinas, " Me voici! ».

Nous présenterons et commenterons en bref les textes qui composent ce recueil. Le premier, de Jean Halpérin, s'intitule "Passé, présent, futur ». Ce texte étonne doublement, d'une part, parce qu'il ne porte pas sur Ricœur, bien que celuici ait considérablement réfléchi à la question du temps, et d'autre part, parce l'auteur adopte une approche plutôt éclectique: il aborde les droits humains, plusieurs termes propres à l'exégèse juive, le socialisme, le développement, la fragilité de l'État d'Israël, dont il voit l'indépendance comme " le début d'une nouvelle et difficile aventure qui interpelle le passé, le présent et l'avenir» (p. 50).

Divisé en trois points, le texte d'Olivier Mongin met l'accent sur la continuité du versant politique de l'ontologie de l'agir que Ricœur déploie inlassablement au plan philosophique. Dans un premier temps, Mongin résume le rapport de Ricœur à la Théorie de la justice de J. Rawls ainsi qu'à la pluralité des biens de M. Walzer dans ses Spheres of Justice. Ce qui ressort de cette discussion avec le libéralisme et le communautarisme, c'est d'abord «le souci jamais démenti de ne pas abandonner la dimension téléologique de l'action (celle du Bien) sans pour autant renoncer à la figure du sujet responsable de ses actes » (p. 56). Ensuite sous la thématique « conviction et 
démocratie ", l'auteur analyse l'opinion droite, la rhétorique et le consensus conflictuel qui caractérisent, chez Ricœur, l'espace public: "Certes la conviction a un rôle décisif, mais elle doit se plier aux règles d'universalité de la discussion parce que les conflits peuvent tourner mal»(p. 74). Dans un dernier temps, Mongin reprend les trois niveaux sur lesquels portent les relations entre l'idéologie et l'utopie, dont nous ne soulignons au passage que la traduction très récente de Ideologia and Utopia au Seuil en mai 1997.

La question du tragique est abordée dans un bref article de Guy Petitdemange. Cette contribution, basée sur quatre extraits de l'œuvre, permet de souligner la place décisive qu'occupe la «non-philosophie » dans la méditation de Ricœur : « Seules les Écritures donnent à sa réflexion autant d'envol» (p. 87). Concluant son texte, l'auteur résume les trois axes vers lesquelles pointent le tragique : il illustre d'abord la limite de la connaissance ; il ouvre aussi sur l'énorme opacité du souffrir et reconduit enfin l'homme à son propre terrain, celui de l'action.

La longue étude suivante, qui s'intitule "L'unité de l'œuvre de Paul Ricœur », n'est malheureusement pas à la hauteur de son titre. On apprendra à la lecture des notes que ce texte d'Alexandre Derczansky s'inspire en fait d'un entretien accordé par Ricœur. Outre un passage consacré à l'identité narrative, ce n'est pas le caractère philosophique de cet article qui impressionne mais le degré de familiarité avec lequel se trame l'ensemble du commentaire. En terminant, au sujet de la continuité de l'œuvre de Ricœur, « il y a une fidélité à une prise de position initiale qui n'a jamais besoin de se réaffirmer » (p. 103), un fil rouge essentiellement religieux, " de l'ordre du mystère, qui serait la vérité de toute cette quête" (p. 129).

"Attestation et élection », de Marc Faessler, est à la fois le texte le plus pertinent et le moins pédagogique de ce recueil. Après avoir repris les critiques sévères de Ricœur à l'endroit de Lévinas, l'auteur expose son but : démontrer « que la notion d'attestation mise en avant par Ricœur appelle son fondement dans celle d'élection élaborée par Lévinas» (p. 134). Résumons l'idée. Si Ricœur manque la fondation d'élection, c'est qu'il persiste à voir dans l'absolue Extériorité de l'autre un effet de rupture qui évacue tout accueil de l'autre en soi. Tandis que ce serait faire violence à Lévinas que de rapporter l'élection d'autrui au registre d'une ontologie de l'attestation, comme le fait Ricœur avec la reconnaissance herméneutique de l'Autre dans le Même. Aux dires de Faessler, "l'élection, au sens où Lévinas l'entend, origine éthiquement de l'attestation de soi, mais ne se confond pas avec elle. En revanche [...] l'attestation, au sens où l'explicite Ricœur, est conduite au déploiement de l'herméneutique du soi à partir d'une conscience désormais orientée vers la justice par l'élection du sujet » (p. 146).

Comme l'article précédant, «Vers une herméneutique du soi : la voie longue et la voie courte" de Jean Greisch porte sur Soi-même comme un autre. Cette étude savante se présente de deux façons : elle se veut d'abord une lecture à rebours de l'ouvrage, mais aussi un " débat » entre l'herméneutiques du soi de Ricœur et celle de Martin Heidegger. La lecture à rebours proposée par Greisch cherche à savoir si le qualificatif « herméneutique » s'applique à Soi-même comme un autre, étant donné "le caractère fortement transatlantique de l'ouvrage" (p. 155). À maintes reprises, l'auteur répondra par l'affirmative, notamment en montrant que le mariage entre l'herméneutique et la philosophie analytique en est un de raison (p. 162). En ce qui à trait à l'analyse de l'herméneutique de la facticité chez le jeune Heidegger, Greisch fait preuve d'une connaissance remarquable des textes. Malheureusement, la 
rencontre ou le débat entre les herméneutiques du soi, la voie longue de Ricœur et la voie courte de Heidegger n'aura jamais lieu.

Le dernier texte est consacré à la psychanalyse. Le plus admirable dans l'article de René Major, c'est le souci de situer le contexte dans lequel l'Essai sur Freud (Seuil, 1969) a été accueilli. "En se donnant comme tâche la constitution d'une épistémologie », Ricœur montrait que la psychanalyse «n'est pas une technique [car] son travail se situe essentiellement dans le langage»(p. 176). Ainsi, il y a bien deux conceptions très différentes de la discipline : "La première se refuse à toute herméneutique [...]. La seconde se renforce de la méthode herméneutique qui consisterait en une maîtrise par la conscience de la multiplicité du sens » (p. 179).

Ce livre inclut des repères biographiques ainsi qu'une bibliographie sommaire et critique de Ricœur. Une note sur les collaborateurs a été ajoutée en annexe. Ce recueil s'adresse en priorité à des "spécialistes " même si, outre l'entretien et l'échange de lettres, seulement trois des sept articles sont vraiment substantiels.

DANIEL DESROCHES

Université Laval 